\title{
Advancing Sensor Technology for Aerospace Propulsion
}

Fernando Figueroa, Test Technology, Propulsion Testing, NASA Stennis Space Center

Carolyn R. Mercer, Optical Instrumentation Technology, Instrumentation and Controls, NASA Glenn Research Center.

To be presented at the International Mechanical Engineering Congress and Exposition, November 17-22, 2002, Hilton/Convention Center, New Orleans, LA

\begin{abstract}
NASA's Stennis Space Center (SSC) and Glenn Research Center (GRC) participate in the development of technologies for propulsion testing and propulsion applications in air and space transportation. Future transportation systems and the test facilities needed to develop and sustain them are becoming increasingly complex. Sensor technology is a fundamental pillar that makes possible development of complex systems that must operate in automatic mode (closed loop systems), or even in assisted-autonomous mode (highly self-sufficient systems such as planetary exploration spacecraft). Hence, a great deal of effort is dedicated to develop new sensors and related technologies to be used in research facilities, test facilities, and in vehicles and equipment. This paper describes sensor technologies being developed and in use at SSC and GRC, including new technologies in integrated health management involving sensors, components, processes, and vehicles.
\end{abstract}

\section{INTRODUCTION}

NASA's mission is to develop revolutionary technologies to explore the Universe and improve the quality of life on Earth. At NASA's Stennis Space (SSC) and Glenn Research (GRC) Centers, the focus is on propulsion technology to support air and space transportation systems. Stennis is NASA's center of excellence for rocket propulsion testing. The center has numerous test stands designed to test the Space Shuttle Main Engine (SSME) and rocket engines and components being developed by industry and government agencies. Glenn is NASA's center of excellence in turbomachinery. Extensive research and development in gas turbine engines for aviation and airbreathing propulsion for space transportation is conducted for use by Government and industry.

The national priority for space transportation is to develop systems that are much more cost effective and safer in order to foster a commercial space economy. To achieve this, aircraft-like operations are necessary, including rapid turn around times and highly reliable systems. Similarly, in aviation the trend is toward more automated maintenance to improve safety and reliability in light of greatly increased rates of air travel. We believe that sensor technology is and will continue to play a major role in achieving these goals, by enabling early warning of engine failures, identifying performance degradation, and flagging maintenance requirements. Together with sensors are the associated technologies of communication, actuators and logic. This paper provides a brief overview of current activities at NASA Stennis and Glenn in developing these technologies. An extensive reference list is included to provide the interested reader with additional sources of information. 


\section{SENSORS}

\section{A. Overview}

Sensors being developed by NASA SSC and GRC fall into roughly five groups: Microelectrical/optical and electronics, fiber optics, thin films, and bulk optics. These technologies have been chosen because of their ability to provide maximum information from minimum measurements, their relative unobtrusiveness, and/or their typical small size and weight. Representative developments in each of these areas will be briefly described here. Developments in acoustics, thermocouples and pressure transducers are omitted from this discussion for brevity.

\section{B. Micro Electrical/Optical and Electronic}

\section{i. Leak Detection}

Hydrogen leak detection is a critical requirement for safe launches. We have developed hydrogen sensors sensitive to concentrations ranging from parts per million to $100 \%$ hydrogen in inert environments. Two designs have been fabricated; one based on a Schottky diode the other on resistance measurement. Both designs complement each other; the diode can be tuned for high sensitivity at low concentrations while the resistor can operate more stably at high concentrations. The diode is comprised of a thin insulator sandwiched between a metal oxide and a silicon substrate generates a current that is sensitive to hydrogen concentrations at its surface. A single micro-package has been built including the Schottky diode, resistor, and a heater to maintain a constant operating temperature. This package has flown twice on the Space Shuttle in the aft main engine compartment, reliably obtaining reasonable data in real time. The data was validated by post-processed analysis of periodically sampled gas. [Hunter and Liu et al, 2001]

\section{ii. High Temperature Electronic Nose}

To produce information, rather than just data, about the health or state of a system it is desirable to measure a variety of gases. To keep installation, maintenance and purchase prices low it is desirable to package the many different sensor types into one package. We are developing a high temperature electronic nose to measure $\mathrm{H}_{2}, \mathrm{O} 2$, $\mathrm{NOx}, \mathrm{CO}, \mathrm{CO} 2$ and hydrocarbons in one, robust package.

The Schottky diode sensor described Section ii can be fabricated with different materials to provide species selective sensing. Hydrocarbon detection has been demonstrated by replacing the silicon substrate with silicon carbide, a high temperature semiconductor, and heating the device to disassociate the hydrocarbon into its constitutive gases.

Oxygen and carbon dioxide sensing is achieved by microfabricating an electrochemical cell over a semiconductor-mounted diaphragm. Cathodes contact the gas under study, and a solid electrolyte generates a quantity of current proportional to the ambient gas concentration

Nitrous oxides, carbon monoxide and carbon dioxide sensors can also be based on microfabricated chemically sensitive resistors doped to absorb the respective gases. Nanocrystalline $\mathrm{SnO} 2$ sensing elements are used to provide long-term stabilty. [Hunter and Neudeck et al, 2001] 


\section{iii. Fluorescence Sensor}

A micro-fabricated waveguide-based fluorescence sensor has been designed using surface-plasmon cross coupling to reject noise, and waveguide entrainment for signal enhancement. A interferometrically-generated grating is created onto a photo-resist layer and then lithographically processed to generate a corrugated surface. A metal layer is deposited onto the surface, forming an uniformly thick corrugated layer that is optically opaque to all wavelengths except the one which generates surface plasmons corresponding the the corrugation period. The surface plasmon crosses the metal layer and generates a photon on the other side which can be detected without interference from background radiation. This sensor can be used to detect combustion gases or gaseous leaks. [Giakos, 2001]

\section{iv. Smart Accelerometers}

Piezoelectric accelerometers have long been used on the Space Shuttle Main Engines for vibration monitoring. We have recently improved these workhorse sensors to enable the self-determination of sensor errors caused by structural damage, ambient temperature changes, and changes in mounting conditions. These diagnostic conditions are detected by using the reverse-piezoelectric effect at regular time intervals, while maintaining the conventional forward-piezoelectric capability for acceleration measurements. A high frequency signal is sent to the accelerometer and the resultant electrical signature is observed. Characteristic patterns in this signature correspond to the diagnostic conditions just listed. Observation of these patterns allow the accelerometer data to be placed in the proper context, e.g. data from a damaged sensor will not be used to abort a mission. [Flanagan et. al. 2001]

\section{Fiber Optics}

\section{i. Fiber Bragg Sensors}

Fiber optic sensors are commercially available to measure strain by means of a grating written axially along the fiber core. The gratings are formed by the optical excitation of dopants within the fiber, typically by illuminating a square wave mask on the fiber or using a sinusoidally varying optical intensity distribution formed by the interference of two plane waves. The grating has the property of reflecting back a narrow spectrum of the relatively broadband light propagating through the fiber. The reflected wavelength is proportional to the grating period, and hence to the length of the fiber portion containing the grating. If the fiber is embedded in or fastened securely onto a material, the reflected wavelength is in turn is directly related to the strain of the material. This strain can be either mechanically or thermally induced; attention must be paid to the particular environment to attribute meaning to the sensor data.

For aerospace propulsion, we are interested in high temperature and dynamic measurements. We have embedded fiber Bragg gratings into polymer matrix composites typical of engine externals and they have survived the $300 \mathrm{C}$ temperatures created by the resin transfer molding process. We plan to use these sensors for process control to monitor the resin distribution, with the added benefit of having instrumented parts that can be used for structural health monitoring. Either passive or active actuation of structures containing an array of these sensors generates characteristic signatures that can be used to determine structural health.[Adamovsky et. al 2000] We have recently compared the performance of Bragg sensors to holographic interferometry (see section II.E.iv.c) and have observed good correlation with the added benefit of being 
sufficiently light and compact for flight applications. [Lekki, et. al. 2002] In addition, we have built a dynamic demodulation system to measure rapidly varying wavelengths, and used it to monitor a pulsed air jet impinging on a fiber diaphragm. [Lekki, et. al. 2001]

\section{ii. Fabry-Perot}

\section{a. LOx Tank Level Sensing}

Measuring the volume of LOX in run tanks posses some difficult problems. The high pressures (up to 8,500 PSI), high flows, cryogenic temperatures, and the danger of contamination make commercial level systems unusable. Various technologies have been explored. One such technology emits a laser beam through a glass window to detect steep density changes as indicators of level; another uses time-domain reflectometry in which light beams travel through optical fibers immersed in the tank and detects temperature changes associated with steep density changes. Implementation of these level measurement systems is complicated by the fact that it is costly and complex to make modifications to existing tanks. Stainless steel rods protecting a column of discrete temperature sensors are being implemented in new tanks to detect the steep change of temperature indicative of the level of LOX. However, these technologies measure fluid level, and LOX, when pressurized by gaseous Nitrogen, presents a dynamic, boiling interface, that will cause large errors.

A new method is being explored whereby the tank is basically weighed using a set of strain gages distributed throughout the support structure of the tank. The challenge with this approach is to be able to measure very small deformations (tens of microstrain) and to eliminate dynamic loading caused by fluid flow and external conditions such as wind. The use of fiber-optic strain gages is also being explored in this approach, since these gages display immunity to electromagnetic noise, and signals can travel long distances by fiber-optic cable with little deterioration.

\section{b. Gas Temperature}

A Fabry-Perot sensor has been developed to measure temperatures ranging from $20 \mathrm{C}$ to 600C. A sapphire fiber delivers broadband light to an etalon fabricated at one end. The gap of the etalon contracts or expands based on the temperature of the fiber tip. Multi-wavelength interference reflected back from the etalon through the fiber is monitored and used to determine the tip's temperature. This sensor was flown in the nozzle of an OV-10D aircraft to measure exhaust gas temperature. [Tuma et. al. 1999]

\section{Thin Films}

Thin-film technology has advanced to provide multiple measurements in harsh engine environments. Measurements of strain magnitude and direction, heat flux surface temperature and gas velocity have all been demonstrated at temperatures up to $1000 \mathrm{C}$. Thin-film thermocouples sputtered onto Space Shuttle Main Engine turbine blades have provided data at $1100 \mathrm{C}$ in lab tests.

Heat flux is measured by measuring temperatures across an insulating layer. These temperatures are determined by either thermocouples, or more recently, resistors. The resistance method provides higher signal-to-noise, is easier to fabricate, and has a smaller footprint. [Fralick et. al. 2002] Strain measurements have been made on a 
variety of engine components, including compressor and turbine blades, disks, and exhaust manifolds. [Lei et. al. 1998] Work is currently underway to extend the temperature range of both thin-film strain and temperature sensors by using ceramic films.

Gas velocity is measured in two ways. To measure the velocity profile within a boundary layer, a thin-film thermocouple array is used to measure the temperature difference across a thin-film heater strip. To measure mean velocity, thin-film piezoresistors are mounted on a probe inserted into the flow. Velocity is deduced by relating the measured voltage drops to velocity for a given Reynolds number. [Hwang 2002]

\section{E. Bulk Optics}

\section{i. Detection of Hydrogen Fires}

Hydrogen fires are invisible to the naked eye. Therefore, SSC has developed systems to detect these fires. One system can discriminate a hydrogen fire regardless of direct line-of-sight view or indirect reflections from flare stacks. Flare stacks are used to purposely burn hydrogen as measure of safety. Another system is helmet-mounted to help firefighters see hydrogen fires without being encumbered by having to carry additional equipment.

\section{ii. Plume Characterization and Analysis}

Two technologies have been developed since the 1980's. Emission spectroscopy (ES) and absorption spectroscopy (AS).
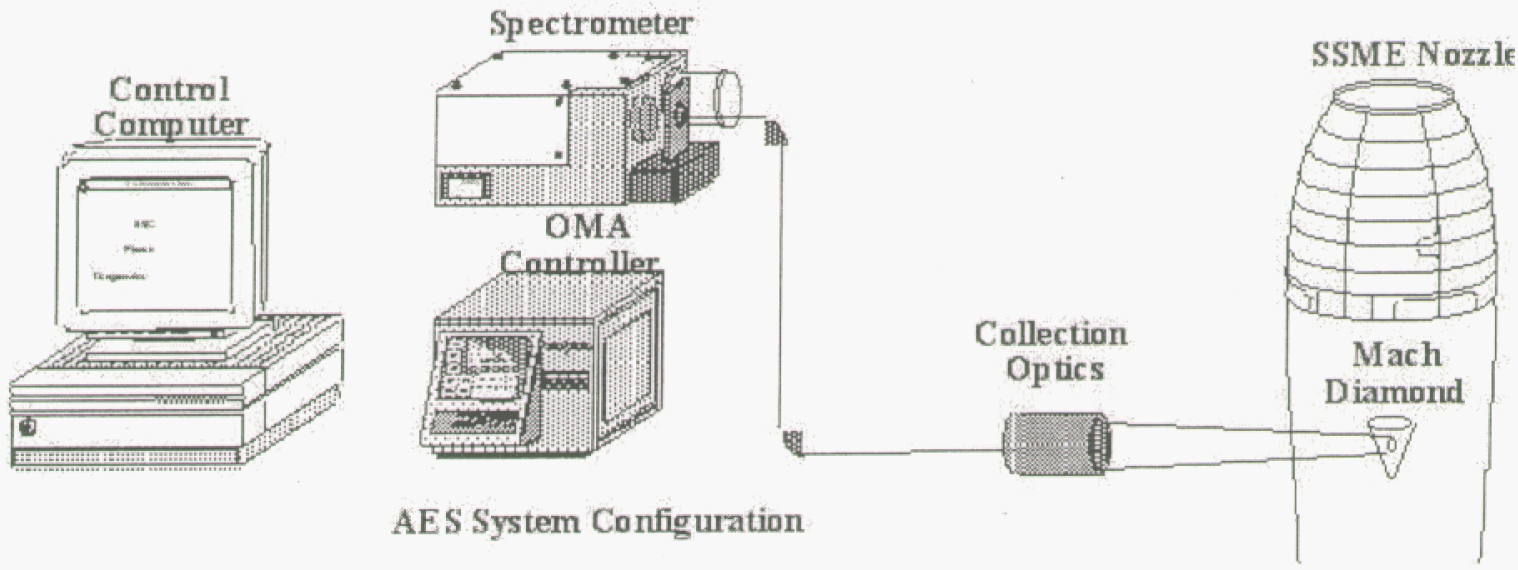

AES System Configuration

\section{Figure 1 Emission Spectroscopy}

\section{Emission Spectroscopy (ES)}

ES has been in use since 1989 to characterize the elements in the SSME [Tejwani et al, 1996]. A schematic of the system is shown in Figure 1. The plume exhibits a bright structure, denominated the Mach Diamond, typified by conditions of high temperature and pressure. A collection optics sub-system is pointed to the Mach Diamond to collect radiation emitted by constituent elements of the SSME as they wear and end up in the plume. An Engine Diagnostics Console (EDC), developed at SSC, identifies and quantifies in parts-per-billion (ppb) elements in the plume. Details of the EDC are available in [Tejwani et al, 1996] and [Bircher et al, 1995]. In the case of the SSME, 
metallic elements that may be present in the plume include $\mathrm{Ni}, \mathrm{Fe}, \mathrm{Cr}, \mathrm{Co}, \mathrm{Mn}, \mathrm{Cu}, \mathrm{Ag}$, $\mathrm{Al}$, and Pd [Tejwani et al, 1996].

Absorption Spectroscopy (AS)

An AS system was developed to be used in situations when the plume does not exhibit a structure at sufficiently high temperatures for ES. A light beam is emitted and received, traveling through the plume near the edge of the nozzle. Absorption of specific light spectral components is used to identify and quantize constituent elements of the SSME. One shortcoming of the AS method is that it captures only information along the optical axis of the beam, but it has good sensitivity detecting erosion upstream of the turbopumps [3].

Fourier Transform Infrared (FTIR) spectroscopic methods are also of interest in engine health monitoring, environment monitoring, and combustion diagnostics. This is a promising technology to be explored in the near future.

\section{iii. Combustion Diagnostics}

Several spectroscopic techniques have been adapted to provide information regarding combustion flows at the high pressures and using the complex fuels typical of hydrocarbon burning jet engines. Among these include the development of planar laser induced fluorescence to identify volumetric chemical species distributions at up to 42 atmospheres, and the concomitant use of chemiluminesence, Mie scattering, Raman spectroscopy and particulate diagnostics to provide qualitative insights into the effect of various combustor and injector designs. Linking burning patternation to the formation of unwanted species such as NOx is one objective of these tests; another is the quantitative determination of soot formation and insight into the performance of active combustion control technologies. [Locke 2000]

\section{iv. Gas Path Measurements}

\section{a. Seeded-flow velocimetry}

NASA has long been in the forefront of flow measurement technologies. One class of instrumentation records the behavior of small particles introduced into flows of interest to determine velocity of the fluid at a point or in a plane. Typically we have been interested in the flow through gas turbine engines. Historically we have used laser Doppler velocimetry to make point measurements of the highly turbulent flows in inlets, compressors, turbines, and nozzles, but of late we rely much more on particle imaging velocimetry (PIV) to capture entire planes of data simultaneously. PIV has been used to measure the tip clearance flow within high-speed compressors, the hot supersonic flow out of an engine nozzle, and numerous other engine flows. Second order statistics in space and time are obtained using fuzzy logic to provide high spatial resolution. [Wernet 2000] Doppler Global Velocimetry, developed by researchers at NASA LaRC, measures the frequency shift of high speed particles without requiring individual particle imaging. This enables the measurement of large areas, and we are now adapting it for fiber optic illumination and detection for the high speed flows encountered in new space transportation systems. 


\section{b. Molecular scattering}

Another class of flow measurement does not require the introduction of particulate to follow the flow, but instead extracts information from laser light scattered directly from the gas molecules present in the flow. Rayleigh scattering has been developed to enable the simultaneous measurement of gas velocity, density, and temperature at either high data rates at a limited number of points, or slower acquisition across a plane. Another application of this technology is the determination of gas mixtures when densities and temperatures are known. This work is typically used to interrogate supersonic, turbulent jets in the pursuit of fundamental understanding of the physics of noise generation. [Seasholtz 2002]

\section{c. Interferometry}

We developed a common-path interferometer that is capable of phase-stepping for increased refractive index measurement accuracy. The device uses a liquid crystal layer sandwiched between electrode-coated glass plates with a single microsphere trapped within the liquid crystals. A collimated beam passes through a flow of interest and is focused onto the microsphere generating a spherical reference wave. This spherical reference wave propagates collinearly with light that passed through the sandwich with its phase information intact, forming an interference pattern on a detector plane. This pattern contains information about the refractive index distribution of the flow, which in turn is related to temperature, density, or species concentrations. The liquid crystals serve to introduce known phase delays into the object beam, enabling the use of phase stepping interferometry algorithms to more precisely and automatically determine the phase distribution. [Mercer, 2002]

\section{v. Surface Measurements}

\section{a. Luminophor-based paints}

Paints containing luminophors that exhibit an optical response that varies with temperature and pressure extensively used to measuring surface pressures and temperatures over entire surfaces at once. The coatings are applied to engine surfaces, then illuminated with a greenish light source to excite a luminescent response typically in the red. Either the intensity or time-rate-of-decay of the luminescence is measured to determine the either the temperature or pressure of a surface. Borescope systems enable measurements in the confined spaces of engine components, and synchronization is used to measure rotating components. Current efforts are underway to increase the response time of the paints for dynamic measurements and to improve high temperature performance. Recent tests have shown survivability at $1200 \mathrm{C}$ for bound phosphors and $1700 \mathrm{C}$ for sputtered phosphors. [Lepicovsky 2002]

\section{b. Pyrometry}

A multi-wavelength pyrometer has been developed to measure surface temperatures when the emissivity of the material is unknown and with improved the accuracy over conventional two-wavelength configurations. Either high spatial resolution point measurements or large-area temperature histograms can be made. The system has been used to measure nanostructured thermal barrier coatings, and has been incorporated into a high temperature probe to measure combustion gas temperatures. [Ng 2001] 


\section{c. Holographic Interferometry}

Light scattered from the surface of vibrating structures can be analyzed holographically to determine structural integrity for the non-destructive evaluation of engine components such as blades, bliscs and casings. Characteristic vibration patterns and resonant frequencies are determined and analyzed by a neural network to determine deviations from a norm. The neural network has been trained to automatically flag damaged parts in real time. "Image folding" is used as a pre-processor to increase system sensitivity. Current efforts are underway to determine a rigorous calibration procedure. [Decker 2001]

\section{AUXILIARY SENSOR HARDWARE}

Smart systems require more than just sensors. Power sources, communications technology, logic and actuators are required to process data and effect change. In addition, to avoid the maze of wires required by centralized archictectures, they require either native wireless ability or on-site electronics to provide local processing and communication.

\section{A. Wireless technology}

Wireless sensors are desirable in that installation and commissioning times are reduced to a minimum. Stennis has developed a wireless temperature network system for thermocouples of type $\mathrm{K}$. The system can have up to four transmitter units and each unit supports four thermocouples and one ambient solid-state temperature sensor. The signals can travel up to 0.5 to 1.0 mile. Each transmitter unit operates on battery power that can last up to 7 days reporting every 30 minutes. GRC and NASA Johnson are also developing low power wireless technology for use with engine flight sensors. Smart sensors have been flown on the Shuttle, and programmable wireless correlators are underdevelopment. [Kiefer, 2002].

Availability of ready wireless sensors is very desirable to support monitoring of parameters that may not have been considered in the initial plan. These sensors make it possible to improve interpretation of test results by quickly augmenting the number or type of parameters being monitored. Wireless sensors also allow extracting information during tests that may be used to fill objectives lateral to the test objectives, such as ambient monitoring, or health monitoring of a particular component.

\section{B. Power sources}

The optical sensors described in Section II require light sources to function. Most of these sensors use lasers, laser diodes, superluminescent laser diodes, or light emitting diodes but some require broadband optical radiation. Even narrow band sensors can use broadband light sources as part of a wavelength division multiplexed system.

We are developing a true white "light bulb on a chip" by microfabricating a coiled tungsten filament over a silicon aperture. The filament is heated to produce blackbody radiation. The entire chip assembly will be vacuum sealed to increase the lifetime. To date the unpackaged filament has operated for over 2000 hours and is still running. [Tuma et al 2000]

We also explored the idea of using combustion radiation as a light source. The spectral distribution of typical combustion gases was determined for possible use as a readily available source. 


\section{Electronics}

Because of the high temperatures inherent in aerospace propulsion environments, we have developed significant expertise in Silicon Carbide technologies for sensors and electronics. $\mathrm{SiC}$ operates as a semiconductor at higher temperatures than either silicon or silicon-on-insulators. It also has a high thermal conductivity, making it an excellent choice to fabricate sensors and integrated electronics for high temperature applications. Single crystal, impurity-free wafers are required to produce high quality electronic circuits, and these are not commercially available. Recent breakthroughs in "web growth" processing enables atomically-flat growth over areas as large as a couple of millimeters. This work promises to enable integrated electronics, communications and sensors on one chip. [Neudeck 2002]

\section{Actuators}

We are developing optical actuators for engine component control, particularly for use in explosive-prone environments like fuel systems. Macro actuation has been effected optically by using photodetectors to drive electric motors, but optics can also be used directly. Focused light can be used to optically heat shape memory alloys to either drive mechanical systems or to open switches enabling the redirection of hot gases or fluids for pneumatic or hydraulic control. [Baumbick 2002] Light can also be used to produce surface micro-actuation by illuminating photorefractive materials with interference patterns. These micro-deformations can be used for flow control by tripping boundary layers, for example. [Curley 2000]

We are also developing MEMS-based micro-actuator concepts for use in smart fuel injectors for active combustion control. Small amplitude fuel modulation can effect large changes in combustion efficiency. Shape memory alloys are being considered for lower temperature applications such as fluid control.

\section{HEALTH MANAGEMENT SYSTEMS}

\section{i. Intelligent Integrated Health Management}

Work in this area is defined to reflect the NASA wide effort denominated Integrated Vehicle Health Management (IVHM). The paradigm for IIHM is to develop systems with components defined as "intelligent agents." Each sensor, pump, tank, process, etc. is an intelligent agent capable of monitoring its own health in the context of other components associated with the same process. For instance, a temperature sensor and a pressure sensor monitoring the liquid flowing inside a section of pipe must interpret and monitor their own signals in the context of the laws of physics governing the flow process. The objective is to design high-integrity systems with the ability to determine the health-condition of each element, and the quality of the data produced by the system. Such systems will increase safety; decrease costs of installation, maintenance, commissioning, and operation; and decrease turn-around times from one test-program to the next. 


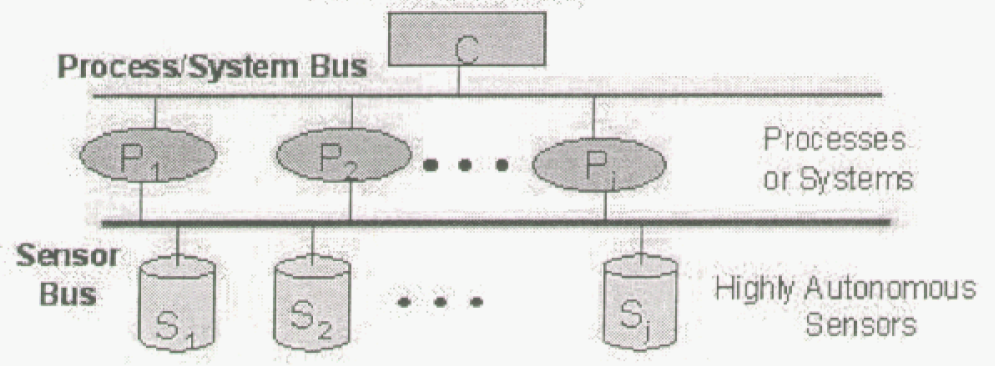

Figure 2 Physical Protocol for IIHM

In order to implement the paradigm, a bus-based protocol is needed, since information must travel to/from sensors to/from processes, to/prom subsystems, and to/from an overall controller [Figueroa and Mahajan 1994; Mahajan and Figueroa, 1995; Figueroa, 2001; Figueroa et al, 2001; Yuan and Figueroa, 2001]. Figure 2 shows a schematic of the physical protocol.

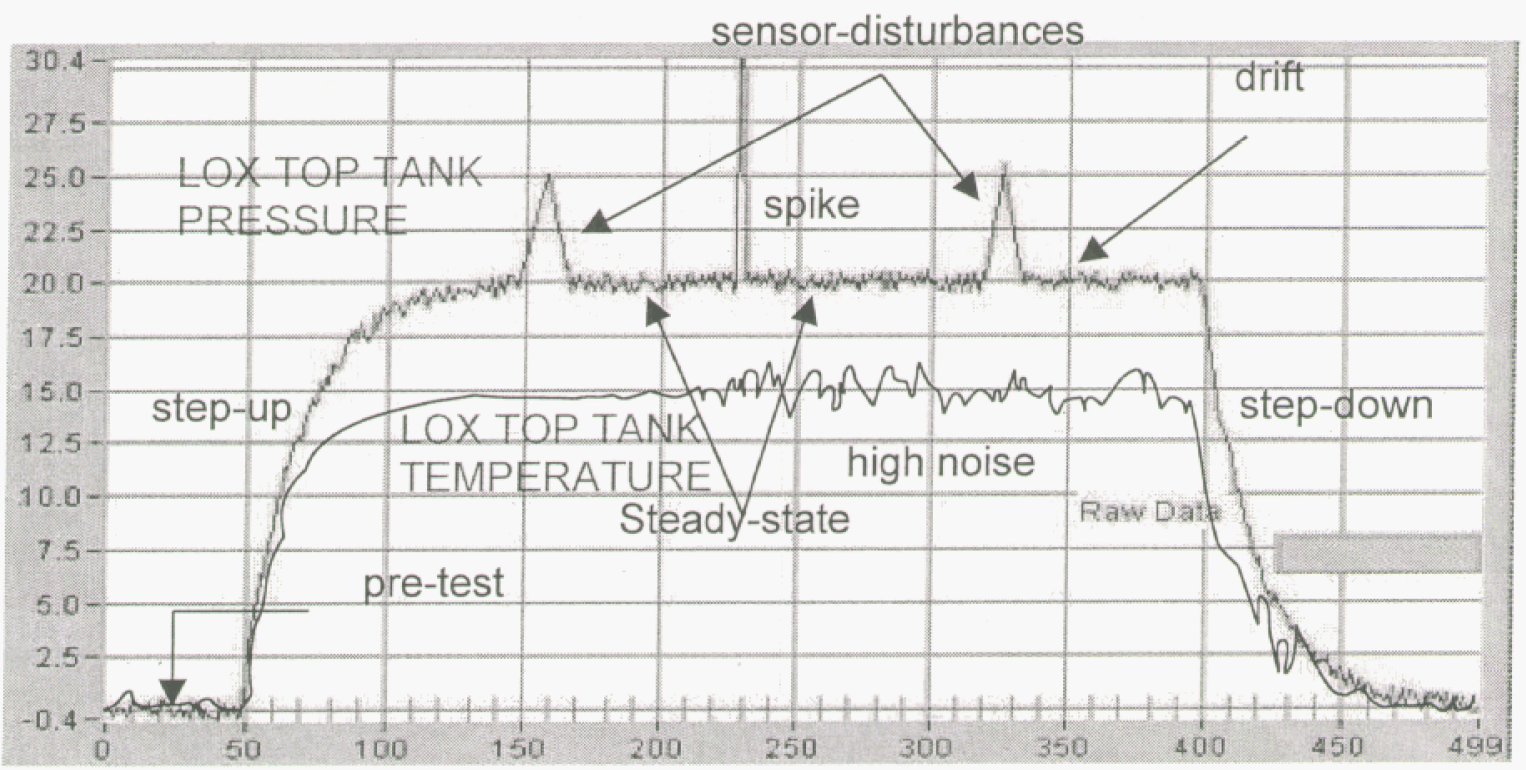

Figure 3 Intuitive Behavior Description

Sensor readings are usually not scrutinized as a matter of "normal" operating procedure. As long as the sensor's "in-calibration" period has not expired, it is assumed to be operating correctly. Its readings may be scrutinized when an anomaly is discovered in the system being monitored by the sensor. Addressing this issue, at the basic level, IIHM focuses on modeling sensors as intelligent (highly autonomous) agents that operate as elements of a distributed network of sensors, processes, and controllers. A modeling method will be used to embed into any sensor the ability to monitor its healthstatus and the quality of its readings. The sensor must extract qualitative behaviors from the data it reads. Hence, each sensor will provide a qualitative description, in time, of the behaviors experienced by the parameter it monitors (e.g. tank pressure step change) 
and any extraneous perturbations (Figure 3). Even though there are many commercial trending software packages, none is capable of describing trends as qualitative behaviors where the key features are shapes of a signal, regardless of the values. The ability to extract these qualitative behaviors is a very powerful tool for monitoring and diagnosis since it mimics how expert operators perform these tasks. With all sensors in a system modeled as highly autonomous, a fusion method at a high-qualitative level will be implemented for monitoring and diagnostics.

An important technological hurdle to implement IIHM resides with the software environment. Such environment must support real-time management of information in many forms, namely analytical, quantitative, and qualitative. It must also provide flexibility for expansion of the system as a natural evolution with time and complexity. In essence, it must combine classic computational tools with artificial intelligence tools and information sharing tools (communications).

\section{ii. Sensor Architectures for Health Management}

To fully realize the benefits that sensors can bring to smart systems, the system (vehicle, engine, etc...) should be designed concurrently with health management hardware and software. Sensor and actuator hardware can be designed to improve system reliability, cost and weight by monitoring processes that have heretofore been left for open loop operation. One example is monitoring compressor or turbine blades in pumps and turbomachinery to detect damage before it becomes safety critical. Another is monitoring tip clearance flows to detect increased leakage, then peforming microactuation to dynamically close the gap. These sensors and actuators should be embedded for maximum performance, so their properties must be considered when designing the shape and materials of engine components. In addition, the sensor and actuator hardware shouldn't be centrally controlled, or the in-system communications links would be too cumbersome. Instead, a well designed system that pushes decision making down to the lowest possible level would provide the most benefit. This places requirements on the development of high temperature sensors, actuators, communications and electronics as discussed throughout Sections II and III above. This "local area node" concept is shown in Figure 4, where local nodes perform all local functions, then are grouped with other nodes to form areas of control, and finally the areas are grouped to form either regions or full systems. 


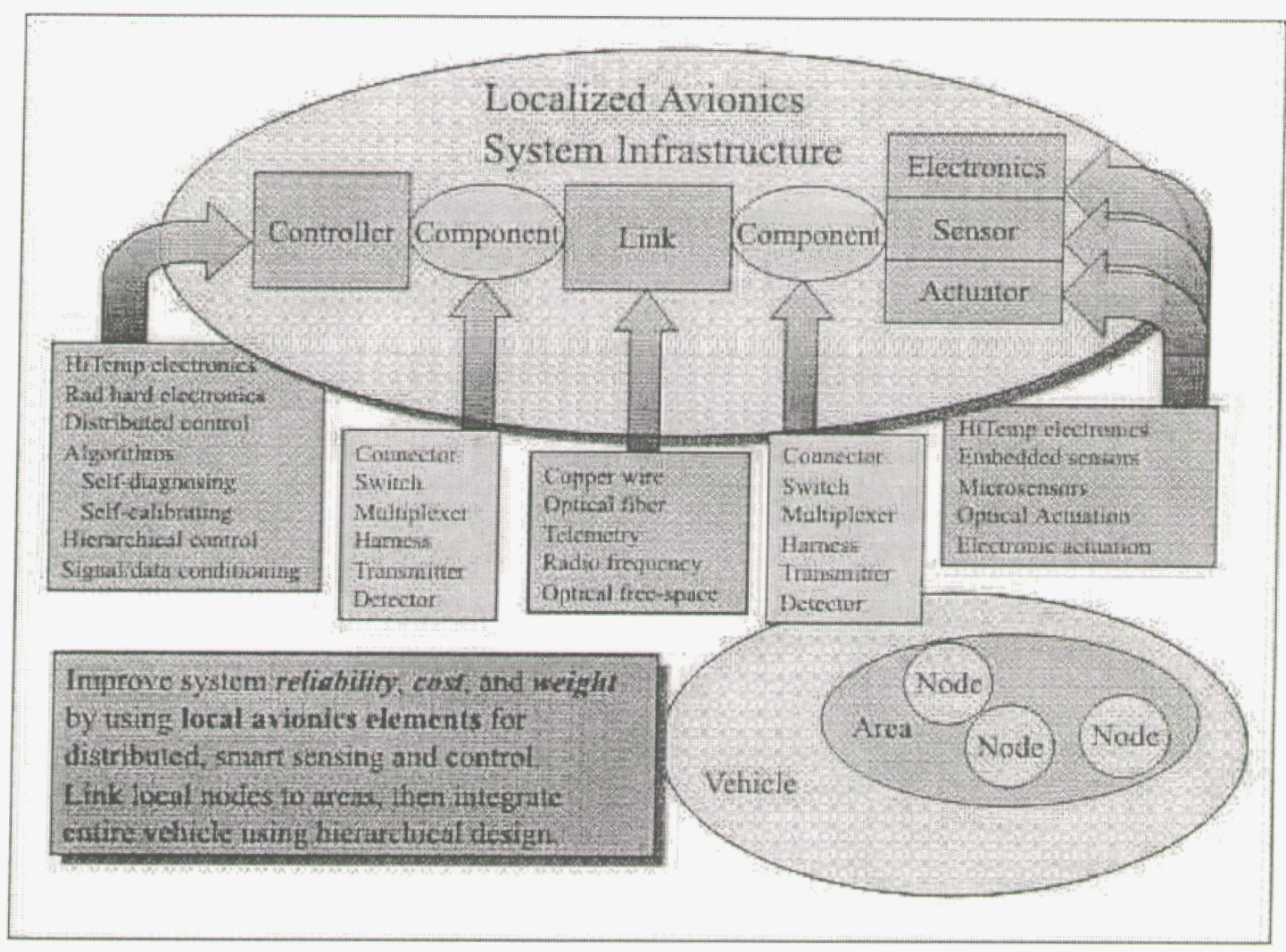

Figure 4. Hardware-based architecture for smart systems

\section{SUMMARY}

Sensor technology is recognized by NASA as a vital element of intelligent systems. This paper briefly describes recent work at the NASA Stennis Space and Glenn Research Centers to develop sensor technology for aerospace propulsion systems. We anticipate growth in this area as future aerospace transportation systems incorporate intelligence to meet increased demand for low cost development and operations and increased safety.

\section{ACKNOWLEDGMENTS}

The authors would like to thank the following people for useful discussions and the references used in this paper: Mr. Tim Bencic, Dr. Arthur Decker, Mr. Gus Fralick, Dr. Jih-Fen Lei, Mr. John Lekki, Dr. Randy Locke, Dr. Larry Matus, Dr. Robert Okojie, Dr. Richard Seasholtz, Dr. Margaret Tuma, and Dr. Mark Wernet, and especially Dr. Grigory Adamovsky and Dr. Gary Hunter for continued conversations about the benefits of smart systems and the potential for sensor technology to change the future. Our thanks also to Mr. Gregory McVay, and Dr. Gopal Tejwani for enlightening our understanding of engine plume diagnostic methods, and Dr. William St. Cyr for valuable suggestions to improve the manuscript.

\section{REFERENCES}

Fernando Figueroa and H.R. Everett, Handbook of Mechatronics, Section on "Distance Measuring and Proximity Sensors", CRC Press, to be published on February 2002. 
G. D. Tejwani, F. E. Bircher, D. B. Van Dyke, G. P. McVay, C. D. Stewart, L. A. Langford, and C. C. Thurman, "Space Shuttle Main Engine Exhaust-Plume Spectroscopy," Spectroscopy V. 11, pp. 31-43, 1996.

F. E. Bircher, G. D. Tejwani, D. B. Van Dyke, and C. C. Thurman, "Engine Diagnostic Console: Recent Development and Test Results with SSME Data," AIAA 95-2785, 31 ${ }^{\text {st }}$ AIAA/ASME/SAE/ASEE Joint Propulsion Conference and Exhibit, San Diego, CA, July 10-12, 1995.

Gregory P. McVay, Felix E. Bircher, Gopal D. Tejwani, Samuel E. Stephens, David B. Van Dyke, and Charles C. Thurman, "Development of an Atomic Absorption System for the Space Shuttle Main Engine," AIAA 97-2665, 33 ${ }^{\text {rd }}$ AIAA/ASME/SAE/ASEE Joint Propulsion Conference \& Exhibit, Seattle, WA, July 6-9, 1997.

Fernando Figueroa, "Facility Monitoring: A Qualitative Theory for Sensor Fusion," AIAA/ASME/ASEE Joint Propulsion Conference and Exhibit, 8-11 July, Salt Lake City, Utah, US.

Figueroa, Fernando; Schmalzel, John; Solano, Wanda; and Thurman, Charles, 'A Future Visison of Data Acquisition - Distributed Sensing, Processing, and Health Monitoring," IEEE Instrumentation and Measurement Technology Conference, Budapest, Hungary, May 21-23, 2001, pp 486-489.

Fernando Figueroa and Xiaojing Yuan, "Sensor Fusion for a Network of Processes/Systems with Highly Autonomous Sensors," IEEE International Workshop On Virtual And Intelligent Measurement Systems, Budapest, Hungary, May 19-20, 2001, pp 4-10.

Fernando Figueroa and Ajay Mahajan, "Generic Model of an Autonomous Sensor," Mechatronics, Vol. 4, No. 3, pp. 295-315, 1994

Ajay Mahajan and Fernando Figueroa, "Dynamic Across Time Autonomous - Sensing, Interpretation, Model Learning, and Maintenance Theory," Mechatronics, Vol. 5, No. 6, 1995, pp. 665-693.

Adamovsky, Grigory, Lekki, J., Sutter, J.K., Sarkisov, S.S., Curley, M.J., Martin, C.E., "Smart Microsystems with Photonic Element and their Applications to Aerospace Platforms," SPIE Symposium on Smart Materials and MEMS, Smart Structures and Devices Conference, Melbourne Australia, December 13-15 (2000)

Baumbick, Robert J. "Shape Memory Alloy Actuator," US Patent \#6,367,250 April 9, 2002.

Curley, Michael J., Fields, Aisha, Sarkisov, Sergey S., Kukhtarev, Nikolai V., Kulishov, Mykola, Adamovsky, Grigory, "Optical actuators based on photorefractive materials controlled by moving holographic gratings" Organic Photorefractives, Photoreceptors, and Nanocomposites, Keith L. Lewis, Klaus Meerholz, Editors, Proceeding of SPIE Vol. 4104 (2000) 
Decker, Arthur J., "Optimization of Training Sets for Neural-Net Processing of

Characteristic Patterns from Vibrating Solids" NASA/TM--2001-210979

$46^{\text {th }}$ Annual Meeting, International Symposium on Optical Science and Technology sponsored by The International Society for Optical Engineering

San Diego, CA, July 29-August 3, 2001

Flanagan, Patrick, Lekki, John, "A Self-Diagnostic System for the M6 Accelerometer" Joint Propulsion Conference Salt Lake City, UT, July 8-11, 2001 Publisher/Sponsor AIAA, ASME, SAE, ASEE

AIAA 2002-3318

Fralick, Gus, Blaha, Charles, Wrbanek, John, "Thin Film Heat Flux Sensor of Improved Design" ISA 48th International Instrumentation Symposium, San Diego, CA May 6-10,2002.

Giakos, George C., Meehan, K., Tuma, M., "Exploitation of Enhanced Fluorescence via Cross Coupling Principles towards the Design of an Optical Integrated Thin-Film Sensor for Nanotechnology and Biomedical Applications" IEEE instrumentation and Measurement Technology Conference Budapest, May 21-23, 2001

Hunter, Gary W., Neudeck, Philip G., Fralick, Gustave, Makel, D., Liu, C.C., Ward. B., Wu, Q.H., Thomas, Valerie, Hall, G, "Microfabricated Chemical Sensors for Space Health Monitoring Applications," AIAA Space 2001 Conference, Albuquerque, NM, August 28-30, 2001 AIAA \#2001-4689

Hunter, Gary W., Liu, C.C., Makel, D.B., "Microfabricated Chemical Sensors for Aerospace Applications," MEMS Handbook, CRC Press LLC, ed. M. Gad-el-Hak, Ch. 22. (2001)

Hwang, Danny P., Fralick, Gustave C., Martin, Lisa C., Wrbanek, John D., Blaha, Charles A., "An Innovative Flow-Measuring Device: Thermocouple Boundary Layer Rake," AIAA 40 ${ }^{\text {th }}$ Aerosciences Meeting and Exhibit, Reno, NV, January 14-17 (2002) AIAA \#2002-0289

Kiefer, Karl F., "Wireless Sensors - Milestones and Stumbling Blocks," Society for Experimental Mechanics IMAC-XX: Conference on Structural Dynamics, Los Angeles, CA, February 4-7, 2002

Lei, Jih-Fen, Will, Herbert A, "Thin-Film Thermocouples and Strain-Gauge Technologies for Engine Applications," Sensors and Actuators A, Vol 65, pp 187-193 (1998)

Lekki, John, Adamovsky, Grigory, Floyd, Bertram, "Demodulation system for fiber optic Bragg grating dynamic pressure sensing" SPIE's $8^{\text {th }}$ Annual International Symposium on Smart Structures and Materials Conference: Sensory Phenomena Newport Beach, CA, March 4-8, 2001.

Lekki, John, Adamovsky, Grigory, Flanagan, Patrick, Weiland, Ken, "Evaluation of Mechanical Modal Characteristics Using Optical Techniques" SPIE $9^{\text {TH }}$ Annual 
International Symposium on Smart Structures and Materials Conference: Smart Structures and Integrated Systems San Diego, CA, March 17-21, 2002

Lepicovsky, Jan, Bencic, Timothy J, "Use of Pressure Sensitive Paint for Diagnostics in Turbomachinery Flows with Shocks," ISABE-2002

Locke, Randy J., Hicks, Yolanda R., deGroot, Wilhemus A., "Non-Intrusive, LaserBased Imaging of Jet-A-Fuel Injection and Combustion Species in High Pressure, Subsonic Flows," $25^{\text {th }}$ Airbreathing Propulsion Subcommittee Meeting sponsored by the Joint Army-Navy-NASA-Air Force, Monterey, CA, November 13-17, 2000

Mercer, C. R., Raman, G, "Quantitative Interferometry in the Severe Acoustic Environment of Resonant Supersonic Jets" AIAA Journal, Volume 40, Number 2, Pages 438-442

Neudeck, Philip G. Powell, J. Anthony, Trunek, Andrew, Spry, David, Beheim, Glenn M., Benavage, Emye, Abel, Phillip, Vetter, William M., Dudley, Michael, "Homoepitaxial 'Web Growth' of SiC to Terminate C-Axis Screw Dislocations and Enlarge Step-Free Surfaces," Materials Science Forum Vols. 389-393, pp 251-254, 2002.

Ng, Daniel, Fralick, Gustave, "Use of a Multiwavelength Pyrometer in Several Elevated Temperature Aerospace Applications" Review for Scientific Instruments, Volume 72, Number 2, February, 2001

Seasholtz, Richard G., Panda, Jayanta, Elam, Kristie A., "Rayleigh Scattering Diagnostic for Measurement of Velocity and Density Fluctuation Spectra" $40^{\text {th }}$ AIAA Aerospace Sciences Meeting and Exhibit Reno, NV, January 14-17, 2002 AlAA--20020827

Tuma, Margaret L., King, Kevin, Kim, Lynn, Hansler, Richard, Jones, Eric, George, Thomas, "MEMS Incandescent Light Source" SPIE Annual Meeting San Diego, CA, July 30-August 4, 2000 Session: Photonics for Space Environments VII (AM106)

Tuma, Margaret L., Sawatari, Takeo, Lin, Yuping "Flight Tests Performance of an Optical Temperature Sensor with Fiber-Optic Signal Transmission" Instrument Society of America, Albequerque, NM, May 1-6, 1999

Wernet, Mark P., "Application of DPIV to study both steady state and transient turbomachinery flows," Optics \& Laser Technology Vol 32, pp. 497-525 (2000) 